\title{
Protobothrops mangshanensis bite: first clinical report of envenoming and its treatment
}

\author{
Jiri Valenta, Zdenek Stach, Michal Otahal
}

\begin{abstract}
Aim. This case report presents envenoming by the Chinese pit viper Protobothrops mangshanensis (formerly Zhaoermia) and its treatment.

Methods. A 38 year old snake breeder suffered two-fang bites to elbow by a Chinese pit viper Protobothrops mangshanensis resulting in local edema of the affected arm. No other signs of envenoming appeared. On the $5^{\text {th }}$ day following the bite a hematoma developed on the other arm which had been mechanically injured 14 days before. Laboratory testing revealed severe coagulopathy with hypofibrinogenemia and immeasurably prolonged coagulation times.

Results. As substitution therapy with fibrinogen and fresh frozen plasma was unsuccessful and specific antivenom is not produced, antivenin against some other Asian pit vipers GREEN PIT VIPER ANTIVENIN, Thai Red Cross, Thailand was applied. Three doses of antivenom reversed the course of the hemocoagulation disorder.

Conclusion. The case confirms the persistence of active venom components affecting coagulation, difficulty in ameliorating the hemocoagulatin disorder caused by snake venom through substitution therapy and the effectiveness of delayed treatment using antivenin. It points out the potential risk of a clinically asymptomatic progress of envenoming by snake venoms containing hemocoagulation acting components, if the hemocoagulation disorder is not investigated and suitably treated. Therapy using the GREEN PIT VIPER ANTIVENIN, Thai Red Cross, Thailand in this case of envenomation by a Protobothrops mangshanensis bite proved to be applicable and the antivenom could be characterised as a paraspecific active.
\end{abstract}

Key words: snakebite, coagulopathy, afibrinogenemia, chinese pit viper, Protobothrops mangshanensis

Received: August 25, 2011; Accepted with revision: February 9, 2012; Available online: April 19, 2012 http://dx.doi.org/10.5507/bp.2012.021

Department of Anesthesiology and Intensive Care, First Faculty of Medicine, Charles University in Prague and General University Hospital in Prague, Czech Republic

Corresponding author: Jiri Valenta, e-mail:valenta@vfn.cz

\section{INTRODUCTION}

Protobothrops mangshanensis (Mangshan/Mount Mang/Chinese pit viper; local name: Mangshan Laotietou) is a rare endemic Asian pit viper. Initially classed with the Trimeresurus genus and subsequently Ermia and Zhaoermia genera, just a few hundred wild examples of this species exist throughout the locality of Mt Mang in Hunan Province, China. Given its attractive look and due to conservation efforts, this snake is occasionally found in captive reptile collections. Its habitus makes the species one of the largest members of pit vipers.

Although the venom components of pit vipers, as well as symptomatology and therapy of envenomation following a bite from other common pit vipers are known and have been recorded to a sufficient extent, this is the first description of envenoming by a Protobothrops mangshanensis bite.

\section{CASE REPORT}

A Czech snake breeder, a 38 year old man, suffered two fang bites to the elbow of his right arm by an adult specimen of Chinese pit viper Protobothrops mangsha- nensis. His medical history was unremarkable except for a polyvalent allergy (grass, mango, and antibiotics, incl. anaphylactic shock).

A minor painful edema was produced at the bitten site, with no early symptoms of systemic envenomation appearing. On day 2, there was progression of edema in the right upper limb, still without any systemic symptoms of envenomation. On day 3 , the edema was regressing, although hematoma was evident at the bitten site.

On day 5 following the bite, normal movement of the man's left arm, on which there was a musculus biceps injury that the person had suffered 14 days earlier, resulted in rapid development of hematoma at the site of this injury, without any other signs of bleeding noticeable. For these reasons, the man visited the surgical department. Laboratory testing revealed severe coagulopathy with hypofibrinogenemia and prolonged PT and APTT. The patient was sent for hospitalization in the hematology clinic.

A hemocoagulation tests showed that coagulation times were extended to immeasurable values (INR $>10$, APTT >180 s, TT > $180 \mathrm{~s}$ ), in addition, hypofibrinogenemia (FBG $0.6 \mathrm{~g} / \mathrm{L}$ ) and elevation of D-dimers (D-dim, $1594 \mu \mathrm{g} / \mathrm{L}$ ) were found. Blood count and biochemical tests were discovered to be within normal range; myoglobin was $39 \mu \mathrm{g} / \mathrm{L}$ (normal value $<92 \mu \mathrm{g} / \mathrm{L}$ ). $8 \mathrm{~g}$ of FBG 
as well as $1200 \mathrm{ml}$ of fresh frozen plasma (FFP) were administered, without an effective stable rise in FBG levels. On day 6, following consultation with the Toxinology Center, the patient was transferred to that facility for further treatment.

No signs of organ involvement were present on admission. Laboratory tests found afibrinogenemia despite the previous high FBG substitution and coagulation times were prolonged to immeasurable values (Table 1). Antivenom was indicated due to the severity of the results, despite the polyvalent allergy and post-bite delay of five days. As no specific antivenin is produced, the treatment started after antihistaminic premedication (hydrocortisone, bisulepine) with the type specific for Trimeresurus albolabris, and paraspecific for some other Trimeresurus pit vipers: GREEN PIT VIPER ANTIVENIN, Thai Red Cross, Thailand.

Activating clotting time (ACT) was applied for bedside orientation hemocoagulation monitoring, the initial value equaling $204 \mathrm{~s}$. The first dose of the antivenin and substitution of $600 \mathrm{~mL}$ of FFP and $2 \mathrm{~g}$ of FBG caused ACT to decrease to $133 \mathrm{~s}$, despite progression of the post-injury hematoma and edema of the left upper limb occurring clinically. For this reason, a second vial of antivenin was administered. Repeated laboratory tests showed persisting hypofibrinogenemia, prolonged coagulation times and elevated D-dim (FBG $0.13 \mathrm{~g} / \mathrm{L}$, INR 1.45, APTT 43.1 s, TT 78.7 s, D-dim > $6400 \mu \mathrm{g} / \mathrm{L})$. With the continuing prolongation of ACT (178 s), in spite of an additional substitution of $1200 \mathrm{~mL}$ of FFP, a third vial of antivenin was administered. The following examination recorded decreased D-dim $(2560 \mu \mathrm{g} / \mathrm{L})$ and normalized coagulation times (INR 1.1, APTT $29.4 \mathrm{~s}$, TT $22.7 \mathrm{~s}$ ), with a parallel increase in FBG $(0.69 \mathrm{~g} / \mathrm{L})$ (Table 1$)$. The patient showed no signs of hematoma enlargement. For the risk of thrombotic complication, antithrombotic prevention was initiated with unfractioned heparin, $5000 \mathrm{IU} / 24 \mathrm{~h}$ by continuous infusion.

Local findings on both arms had stabilized, without evidence of compartment syndrome. The myoglobin level remained within normal values. The level of platelets and antithrombin activity continued to show no significant reduction in the course of the envenomation (Table 1).

Having undergone follow-up laboratory tests with stabilized values of FBG, hemocoagulation times and no signs of envenoming in progress, the patient was discharged home on day 7. Continued antithrombotic prevention (LMWH - Clexane $0.4 \mathrm{~mL}$ s.c. per $24 \mathrm{~h}$ ) over a period of three days was recommended.

\section{DISCUSSION}

Based on the close relation between the Protobothrops mangshanensis and the other members of the core group of Asian pit vipers, it can be assumed that similarities exist in venom composition and clinical effects.

Components acting on fibrinogen, phospholipases A2 (PLA2) with hemorrhagic and myotoxic activity (zhaoermiatoxin) and other enzymatic components have been isolated in the laboratory from Protobothrops mangshanensis (formerly Zhaoermia) venom ${ }^{1,2}$. The approximate $\operatorname{LD}(50)$ of the venom in mice IP was estimated to be $4 \mathrm{mg} / \mathrm{kg}^{1}$.

Pit viper venoms largely consist of components affecting hemocoagulation, i.e. fibrinogen-converting thrombin-like enzymes causing consumption coagulopathy (elegaxobin Protobothrops elegans venom, purpurase Cryptelytrops purpureomaculatus) (ref. ${ }^{3}$ ), fibrino(geno) lytic proteinases (e.g. Protobothrops mucrosquamatus), specific inhibitors of plasmatic coagulation factors with anticoagulation effects (e.g. T. stejnegeri, T. gramineus), PLT activators (e.g. Protobothrops mucrosquamatus), PLT aggregation inhibiting components (e.g. Protobothrops elegans, Trimeresurus jerdonii) (ref. ${ }^{4,5}$ ) and others. The action of these principally results in uncharacteristic hemocoagulation disorders of consumption and defibrination types with or without PLT involvement. Clinically, this is manifest in increased hemorrhaging but can include potential thrombotic complications $\mathrm{s}^{6-8}$.

Local damage of afflicted tissues is caused by a complex of cytotoxic and myotoxic components. These may even cause a certain degree of rhabdomyolysis and are

Table 1. Values of selected parameters of laboratory examination.

\begin{tabular}{|c|c|c|c|c|c|c|c|c|c|c|}
\hline \multirow{2}{*}{ Parameter } & \multirow{2}{*}{$\begin{array}{l}\text { Normal } \\
\text { values, } \\
\text { units }\end{array}$} & $\begin{array}{c}\text { 5th } \\
\text { Day }\end{array}$ & 6th Day & 6th Day & 6th Day & 6th Day & 7th Day & 7th Day & 7th Day & 7th Day \\
\hline & & $21: 29$ & $2: 11$ & $15: 05$ & $18: 41$ & $21: 52$ & $2: 17$ & $6: 09$ & $11: 21$ & $15: 10$ \\
\hline INR & $0.8-1.25$ & $>10$ & 2.64 & 2.19 & 1.45 & 1.2 & 1.1 & 1.08 & 1.1 & 1.13 \\
\hline APTT & $28-40 \mathrm{~s}$ & $>180$ & 49.8 & 45.2 & 43.1 & 46.6 & 29.4 & 31.3 & 32.3 & 28.8 \\
\hline TT & $12-18 \mathrm{~s}$ & $>180$ & 83.7 & 69 & 78.7 & 55.3 & 22.7 & 18.5 & 20.9 & 18.7 \\
\hline FBG & $2.0-4.0 \mathrm{~g} / \mathrm{L}$ & 0.6 & 0.6 & 0 & 0.13 & 0.21 & 0.69 & 1.64 & 0.99 & 1.23 \\
\hline AT (\%) & $70-140 \%$ & 113 & 108 & 112 & 114 & 117 & 110 & 122 & 99 & 112 \\
\hline D-dim & $<190 \mu \mathrm{g} / \mathrm{L}$ & 1594 & 3022 & $>6400$ & $>6400$ & $>6400$ & 2560 & 3834 & 1248 & 1310 \\
\hline Platelets & $142-327 \times 10^{9} / \mathrm{L}$ & 270 & $\mathrm{n} / \mathrm{a}$ & 264 & $\mathrm{n} / \mathrm{a}$ & 147 & $\mathrm{n} / \mathrm{a}$ & 168 & 183 & 194 \\
\hline $\mathrm{ACT}$ & $100-120 \mathrm{~s}$ & $\mathrm{n} / \mathrm{a}$ & $\mathrm{n} / \mathrm{a}$ & 204 & 133 & 178 & 123 & 160 & 145 & 124 \\
\hline Antivenin & No. of vials & & & 2 & & 1 & & & & \\
\hline
\end{tabular}


responsible for necrosis and possible compartment syndrome $^{7-9}$. We cannot exclude effects of circulation-acting components causing hypotension in which may participate hemorrhagins with an extravasation activity ${ }^{7}$.

In the described case, evident coagulopathy with severe laboratory findings developed but until effective therapy with symptoms of bleeding in previously traumatized tissue only. There was classic symptomatology of envenoming by a pit viper, i.e. defibrination syndrome, caused in part by fibrino(geno)lytic components as well as fibrinogen-converting thrombin-like enzymes, the latter supported by findings such as the high D-dim levels recorded. Measurable coagulation times found at undetectable FBG levels (Clauss test) can be explained by the presence of fibrin monomers in plasma resulting from the complex action of venom components.

Effect of antivenom therapy was evident at a relatively rapid normalization of hemocoagulation findings. Spontaneous biodegradation of snake venom hemocoagulation acting components lasts much longer (10-14 days) and wears off very slowly even when using substitution therapy with plasma and concentrates.

The normal antithrombin (AT) levels maintained, may indicate that these enzymes cannot be inhibited by the AT-heparin complex, identical to venoms of other snake genera, such as Echis sp. (ref. ${ }^{10}$ ). Similarly, normal PLT levels during the envenomation displayed evidence of the prevailing influence of PLT aggregation-inhibiting components. Affection by myotoxic PLA2 isolated from the venom of Protobothrops mangshanensis (formerly Zhaoermia) (ref. ${ }^{1,2}$ ) was not shown clinically or in laboratory results.

\section{CONCLUSION}

The case confirmed the long-term persistence of active venom components affecting coagulation in addition to the difficulty to affect the pathological hemocoagulation processes through substitution therapy, as well as attesting to the effectiveness of delayed treatment using antivenom. It points out the potential risk of clinically asymptomatic progress of envenoming by venoms containing hemocoagulation acting components, if the hemocoagulation disorder is not examined and suitably treated.

Therapy using the GREEN PIT VIPER ANTIVENIN, Thai Red Cross, Thailand in the case of envenomation by a Protobothrops mangshanensis (formerly Zhaoermia) bite proved to be applicable and the antivenom could be characterised as a paraspecific active.

\section{ABREVIATIONS}

PT, Prothrombine time; APTT, Activated partial thromboplastine time; INR, International normalized ratio; TT, Thrombin time; FBG, fibrinogen; D-dim, D-dimer; FFP, Fresh frosen plasma; ACT, Activated coagulation time; IU, International units; LD(50), Lethal dose for 50\%; PLT, Platelets; AT, Antithrombine; PLA2, Phospholypasis A2.

\section{REFERENCES}

1. Mebs D, Kuch U, Coronas FI, Batista CV, Gumprecht A, Possani LD. Biochemical and biological activities of the venom of the Chinese pitviper Zhaoermia mangshanensis, with the complete amino acid sequence and phylogenetic analysis of a novel Arg49 phospholipase A2 myotoxin. Toxicon 2006;47:797-811.

2. Murakami MT, Kuch U, Betzel C, Mebs D, Arni RK. Crystal structure of a novel myotoxic Arg49 phospholipase A2 homolog (zhaoermiatoxin) from Zhaoermia mangshanensis snake venom: insights into Arg 49 coordination and the role of Lys 122 in the polarization of the C-terminus. Toxicon 2008;51:723-35.

3. Tan $\mathrm{NH}$.. Isolation and characterization of the thrombin-like enzyme from Cryptelytrops purpureomaculatus venom. Comp Biochem Physiol C Toxicol Pharmacol 2010;151:131-6.

4. Oyama E, Furudate N, Senuki K, Takahashi H. Purification and characterization of a new platelet aggregation inhibitor with dissociative effect on ADP-induced platelet aggregation, from the venom of Protobothrops elegans (Sakishima-habu). Toxicon 2009;53:706-12.

5. Chen Z, Wu J, Zhang Y, Yang X, Yu G, Zhu S, Lee W, Lu Q, Zhang $Y$. A novel platelet glycoprotein Ib-binding protein with human platelet aggregation-inhibiting activity from Trimeresurus jerdonii venom. Toxicon 2011;57:672-9.

6. Chan JC, Kwok MM, Cockram CS, Prematilleke MN, Tomlinson B Critchley JA. Blood coagulation abnormalities associated with envenoming by Trimeresurus albolabris in Hong Kong. Singapore Med J 1993;34:145-7.

7. Warrell DA. Clinical toxicology of snakebite in Asia. In: Meier J, White J, editors. Handbook of Clinical Toxicology of Animal Venoms and Poisons. Boca Raton, New York, London, Tokyo: CRC Press; 1995. p. 493-593.

8. Chen YW, Chen MH, Chen YC, Hung DZ, Chen CK, Yen DH, Huang Cl, Lee $\mathrm{CH}$, Wang $\mathrm{LM}$, Yang CC. Differences in clinical profiles of patients with Protobothrops mucrosquamatus and Viridovipera stejnegeri envenoming in Taiwan. Am J Trop Med Hyg 2009;80:28-32.

9. Rojnuckarin P, Mahasandana S, Intragumthornchai T, Sutcharitchan P, Swasdikul D. Prognostic factors of green pit viper bites. Am J Trop Med Hyg 1998;58:22-5.

10. Mba EC, Onyemelukwe GC. Antithrombin III in Echis carinatus envenomation in northern Nigeria. Acta Haematol 1989;81:98-100. 